\title{
Partisan differences in physical distancing are linked to health outcomes during the COVID-19 pandemic
}

\author{
Anton Gollwitzer ${ }^{1 凶}$, Cameron Martel $\mathbb{B}^{2}$, William J. Brady', Philip Pärnamets $\mathbb{1}^{3,4}$, \\ Isaac G. Freedman $\mathbb{1}^{5}$, Eric D. Knowles $\mathbb{D}^{3}$ and Jay J. Van Bavel $\mathbb{D}^{3,6 \bowtie}$
}

Numerous polls suggest that COVID-19 is a profoundly partisan issue in the United States. Using the geotracking data of 15 million smartphones per day, we found that US counties that voted for Donald Trump (Republican) over Hillary Clinton (Democrat) in the 2016 presidential election exhibited 14\% less physical distancing between March and May 2020. Partisanship was more strongly associated with physical distancing than numerous other factors, including counties' COVID-19 cases, population density, median income, and racial and age demographics. Contrary to our predictions, the observed partisan gap strengthened over time and remained when stay-at-home orders were active. Additionally, county-level consumption of conservative media (Fox News) was related to reduced physical distancing. Finally, the observed partisan differences in distancing were associated with subsequently higher COVID-19 infection and fatality growth rates in pro-Trump counties. Taken together, these data suggest that US citizens' responses to COVID-19 are subject to a deep-and consequential-partisan divide.

$\mathrm{P}$ olitical partisanship has been on the rise in the United States and across the world. People in the political minority are afraid to reveal their political identity ${ }^{1}$, people view the opposing party more unfavourably than ever before ${ }^{2}$, and partisan echo chambers abound both in person and online ${ }^{3-5}$. Partisanship also substantially impacts behaviour ${ }^{6,7}$. For instance, people's choices of where to work, live, and shop are driven by political identity as much as they are by religion ${ }^{8}$; partisan attachments distort memory for political events ${ }^{9}$; and political homophily is as influential as educational homophily in online dating ${ }^{10}$. Furthermore, an estimated 81 million Americans reported arguing about politics with close friends and family after the 2016 election $^{11}$, and 34 million hours of Thanksgiving dinner conversation may have been lost in 2016 due to partisan differences between hosts and guests ${ }^{12}$. Exacerbating these partisan differences, social media discourse has the potential to increase political polarization ${ }^{13,14}$, and slanted news outlets tangibly impact voting decisions ${ }^{15}$. In the current era of polarization, then, differences in partisan identity appear to influence a wide variety of social judgements and behaviours ${ }^{16}$.

The coronavirus pandemic provides a unique test of the limits of political partisanship and identity. Does an international crisis threatening substantial loss of life and prolonged economic hardship bring people together in a bipartisan manner? Or, does even a public health crisis of such great magnitude become subject to partisanship? This is a particularly important issue because partisan polarization during a pandemic could lead people to engage in varying health behaviours ${ }^{17}$. For instance, tuning in to right-wing news (such as Fox News) might communicate very different information about the risks of the pandemic - and how to prevent its spreadthan more left-leaning sources (such as MSNBC). Similarly, political leaders from different parties might communicate different levels of risk or fail to model prudent health behaviours (for example, wearing a mask). These factors could then lead partisans to behave differently during a pandemic, which could in turn have important downstream consequences in terms of infection and mortality.

Numerous polls have found a partisan gap in perceptions of risk and support for preventative health behaviours during the COVID-19 pandemic. For instance, an NBC News/Wall Street Journal poll conducted from 11-13 March 2020 found that $68 \%$ of Democrats reported worrying that someone in their family could catch the virus, compared with $40 \%$ of Republican ${ }^{18}$. Furthermore, nationally representative Gallup polls documented that Republicans were less likely to report practising physical distancing in the previous $24 \mathrm{~h}$ than Democrats (7-23 March 2020: 78 versus $~ 90 \%$ ), and this gap strengthened with time through mid-June (8-14 June: $\sim 48$ versus $\sim 89 \%)^{19}$. Finally, several studies surveying Americans on online data collection platforms have shown that self-identified conservatives are less likely to endorse a variety of physical distancing measures (for example, staying 6 feet away from others, wearing masks) ${ }^{20-22}$. These polls reflect a clear partisan gap in beliefs about the pandemic, but do they translate into real behaviour?

It remains unclear whether these polls reflect genuine partisan differences. Polls have substantial limitations. For instance, self-reported partisan differences may be driven by liberals responding in what they think is a socially appropriate manner, or conservatives responding more honestly ${ }^{23,24}$. Additionally, partisan differences may reflect participants responding in ways they believe align with their political in-group and party leaders-known as political 'cheerleading ${ }^{25}$. And, even when people do express genuine attitudes, these attitudes often do not align with their actual behaviour ${ }^{26}$. To overcome these limitations, we tested whether the documented partisan differences in responding extend to real-world behaviour. 
In the current pandemic, epidemiologists and public health officials have strongly encouraged people to physically distance from one another. Numerous studies have shown that physical distancing effectively stopped the exponential spread of COVID-19 at its onset, preventing the inundation of healthcare providers and saving numerous lives ${ }^{27-30}$. To determine whether physical distancing differs as a function of partisanship, we examined whether the political leaning of over 3,000 counties in the United States is linked to the extent to which residents of those counties practised physical distancing behaviours during the first few months of the pandemic (March-May 2020). Specifically, we analysed the aggregated Global Positioning System (GPS) coordinates of approximately $15 \mathrm{mil}-$ lion people across the United States per day (tracked via smartphone location coordinates) to quantify the degree of physical distancing in each US county. Physical distancing was measured in terms of reduction in general movement and reduction in visiting non-essential services (for example, restaurants). Furthermore, to examine the association between party identity and public health, we tested whether partisan differences in physical distancing mediate the relationship between partisanship and COVID-19 infection and fatality growth rates.

Critically, in testing these questions we anticipated that what could appear to be partisan differences in physical distancing may actually reflect social or economic third variables. For instance, liberal areas in the United States experienced higher levels of COVID19 infections and fatalities at the start of the pandemic (early March 2020) and thus may have perceived COVID-19 as more dangerous and engaged in greater preventative measures ${ }^{31}$. To account for this and other possible explanations, we included numerous covariates in our models when testing partisan differences in physical distancing and health outcomes.

\section{Results}

Validation. We examined people's physical distancing behaviours in 3,025 US counties between 9 March and 29 May 2020. Physical distancing was assessed via counties' percentage reduction in general movement and percentage reduction in visiting non-essential services (for example, barbers, restaurants, clothing stores) relative to the period before COVID-19 struck the United States with force (before 9 March). The two physical distancing variables-general movement (mean $(M)=0.21$, s.d. $=0.19$; counties on average exhibited a $21 \%$ decrease in movement) and visiting non-essential services $(M=0.31$, s.d. $=0.30$; counties on average exhibited a $31 \%$ decrease in visiting non-essential services) - were calculated using approximately 15 million daily smartphone GPS coordinates across the United States (see Methods and Supplementary Information for details).

We first validated the two physical distancing measures by examining whether distancing increased as COVID-19 began to spread across the United States (March to early April 2020) but then decreased as states began to reopen (late April to May 2020). All models reported below are three-level, mixed-effects models where observations are nested within counties and counties within states. For the validation analyses, we report models with random intercepts of county and state and random slopes of linear and quadratic time (fitted using linear and quadratic orthogonal terms) at the state level (see Supplementary Information for more detail). The outcome variables were counties' percentage reduction in general movement and percentage reduction in visiting non-essential services compared to pre-COVID-19 (before 9 March; Supplementary Note 1). Two-tailed statistical tests were used in all analyses.

As expected, general movement and visiting non-essential services reduced as COVID-19 first spread in the United States (early March into early April) and this reduction dissipated as states began to reopen (end of April through May). As seen in Fig. 1, changes in physical distancing over time were characterized by a negative quadratic effect (a convex distribution: unstandardized coefficient
$\left(B_{\text {movement }}\right)=-0.858,95 \%$ confidence interval $(\mathrm{CI})[-0.863,-0.854]$ and $B_{\text {visitation }}=-1.317,95 \%$ CI $\left.[-1.325,-1.309], P<0.001\right)$, and an additional negative linear trend $\left(B_{\text {movement }}=-0.336,95 \%\right.$ CI $[-0.341$, $-0.331]$ and $B_{\text {visitation }}=-0.734,95 \%$ CI $\left.[-0.741,-0.726], P<0.001\right)$. Supplementary Information provides additional details of these and all following analyses.

We conducted additional analyses to further substantiate the validity of our physical distancing measures. As expected, distancing was greater when stay-at-home policies had been implemented $\left(B_{\text {movement }}=0.055,95 \% \mathrm{CI}[0.053,0.057]\right.$ and $B_{\text {visitation }}=0.062,95 \% \mathrm{CI}$ $[0.059,0.065], P<0.001)$; on weekends, when fewer people travel for work $\left(B_{\text {movement }}=0.088,95 \%\right.$ CI $[0.087,0.090]$ and $B_{\text {visitation }}=0.026$, $95 \%$ CI $[0.025,0.028], P<0.001)$; and in higher-income counties, where people are able to work from home $\left(B_{\text {movement }}=0.030\right.$, $95 \%$ CI $[0.027,0.032]$ and $B_{\text {visitation }}=0.041,95 \%$ CI $[0.036,0.046]$, $P<0.001$; Supplementary Tables 19 and 20). The final modelincluding effects of orthogonal linear and quadratic time, weekends, state stay-at-home policy, and county median income-provided a good fit to the average trend in the data (Fig. 1). Model marginal $R^{2}$ was 0.35 for the movement model and 0.37 for the visitation model (Supplementary Tables 19 and 20 and Supplementary Note 3). Together, these results suggest that the geotracking data utilized provide valid indices of physical distancing and allow us to test for differences as a function of political partisanship.

Linking partisanship to physical distancing. We examined whether county-level partisanship is linked to physical distancing. Partisanship was measured in terms of county-level voting gap in the 2016 election-the proportion of total votes for Donald Trump (Republican) minus the proportion of total votes for Hillary Clinton (Democrat). We tested three-level, mixed-effects models with random intercepts of state and county (county nested within state), random slope of partisanship at the county and state levels, random slopes of linear and quadratic time at the state level, and the random slope of the interaction between partisanship and linear time at the state level, when possible (Supplementary Note 4). Fixed effects included the orthogonal time terms (linear and quadratic), partisanship (vote gap), the interactions between partisanship and the two time terms, the weekend indicator, and key covariates likely to be associated with physical distancing, including counties' COVID-19 infections per capita (cumulative; assessed daily), whether a stay-at-home policy was in effect on a specific day, party affiliation of state governor, population density, median income, income inequality, median age, age composition (proportion of population aged $0-17,>65$ or $>85$ years; Supplementary Note 5), race/ethnicity (proportion of population Black, Hispanic and Asian), low access to grocery stores (for example, because of distance or income), number of grocery stores, travel time to work, religiosity, change in life expectancy from 1985 to 2010, and percentage employment. See Methods and Supplementary Information for details, descriptive statistics, and information on variable coding. Indicating that our findings are robust to different models, our results were also consistent when running models without these control variables (Supplementary Tables 22 and 23).

We found that the more a county favoured Donald Trump over Hillary Clinton in the 2016 election, the less that county exhibited physical distancing between 9 March and 29 May 2020. Specifically, for every 1 percentage point increase in vote share for Donald Trump over Hillary Clinton, counties exhibited 0.11 percentage points less physical distancing in terms of reducing their general movement and 0.13 percentage points less physical distancing in terms of reducing their visiting of non-essential services $\left(B_{\text {movement }}=-0.114\right.$, $95 \%$ CI $[-0.140,-0.087]$ and $B_{\text {visitation }}=-0.125,95 \%$ CI $[-0.162$, -0.088 ], $P<0.001$; Table 1; visualization of physical distancing by specific location and partisanship is presented in Figs. 2 and 3). Model marginal $R^{2}$ was 0.46 for the movement model and 0.54 for the visitation model (Supplementary Note 6). 

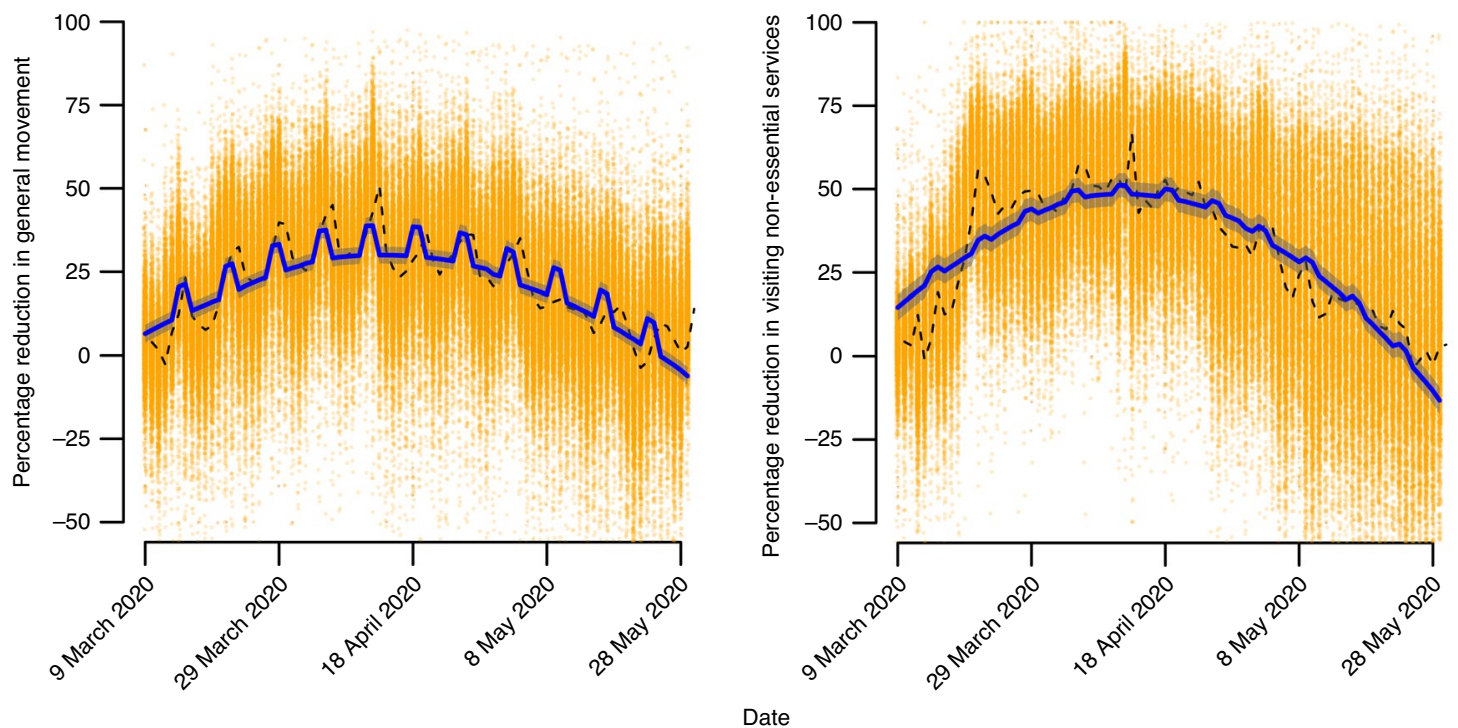

Fig. 1 | Physical distancing as a function of time (9 March to 29 May 2020). Percentage reduction in general movement and visiting non-essential services relative to before COVID-19 (before March 9). In line with the rapid implementation of physical distancing restrictions during mid-March 2020 and then weakening as US states began to reopen, physical distancing increased until early/mid-April and then began to decline. Each point represents one county $(3,025$ counties were included for general movement and 2,073 for non-essential services visitation across 82 days. The total number of data points was 248,050 and 169,890 , respectively). The dashed line denotes the daily average across counties. The blue line represents the average prediction from a multi-level model including the effects of orthogonal linear and quadratic time terms, weekends, state stay-at-home policy, and county median income. Error bands are bootstrapped 95\% prediction intervals (Supplementary Note 2).

Collapsing counties into pro-Trump versus pro-Clinton bins, Trump-voting counties reduced their general movement 9.5 percentage points less and reduced their visiting of non-essential services 19.4 percentage points less than Clinton-voting counties (average reduction, 14.5 percentage points) across the study duration. Illustrating the relative power of the observed links, partisanship was more strongly associated with physical distancing in our main models (when $z$-scoring all the included variables) than any of the other included variables (aside from the time terms, the weekend factor, and median age in the case of visitation; see $z$-scored models in our analysis outputs hosted at https://osf.io/u5pmw/?view only=33f0691a7e694276bef606cb3e22d141;Supplementary Note7). To put this into context, partisanship was more strongly associated with distancing than counties' number of COVID-19 cases per capita, median income, percentage employment, average travel time to work, governor political affiliation, and racial make-up, as well as the other variables noted above.

Additionally demonstrating the robustness of our findings, partisanship was associated with reduced physical distancing even after adjusting for the interactions between each of the included covariates and partisanship (Supplementary Tables 22 and 23), when including in the analyses counties' percentage of employment in various types of profession (for example, agriculture, finance, manufacturing; Supplementary Table 24), when adding specific state policies to the analyses (for example, closing restaurants, closing childcare centres; Supplementary Tables 32 and 33), and when running generalized additive models with time as a smoothed variable (Supplementary Table 25 and Supplementary Note 8).

We next examined how partisan differences in physical distancing changed over time (between March and May 2020). We predicted that the observed differences would decrease as the pandemic worsened and people became more aware of the dangers of COVID-19 (see preregistration of this hypothesis at http://aspredicted.org/blind.php? $\mathrm{x}=\mathrm{n} 84 \mathrm{tw} 2$; Supplementary Notes 9 and 10). We found the opposite pattern. As indicated by negative interaction terms between vote gap and linear time in the models noted above $\left(B_{\text {movement }}=-0.382,95 \% \mathrm{CI}[-0.396,-0.367]\right.$ and $B_{\text {visitation }}=-1.030$, $95 \%$ CI $[-1.054,-1.005], P<0.001$; Table 1), the partisan gap in physical distancing actually increased rather than decreased between 9 March and 29 May 2020, and this was especially true for visiting non-essential services according to model predictions (Fig. 4 and Supplementary Note 11).

We also examined the link between partisanship and physical distancing over time in a different manner. We conducted a series of mixed-effects models to extract the specific coefficients for the link between partisanship and physical distancing (general movement and non-essential visitations, multiplied by 100 to yield percentages) on each day from 9 March to 29 May. All of the covariates noted above were included in these models. We then plotted the extracted coefficients over time (Fig. 5). Linear models predicting these coefficients with orthogonal linear and quadratic time terms ( $z$-scored) indicated that the negative association between pro-Trump voting and physical distancing (collapsed across the two measures) strengthened by approximately -0.047 percentage points per day $(-3.85$ percentage points beteen 9 March and 29 May; linear time term: $B_{\text {movement }}=-0.96,95 \%$ CI $[-1.15,-0.77]$ and $B_{\text {vistation }}=-1.30,95 \%$ CI $\left.[-1.60,-1.00], P<0.001\right)$. We also observed a quadratic pattern for general movement. Pro-Trump voting increasingly linked to decreased physical distancing as the pandemic progressed, finally peaking in early May and beginning to weaken slightly thereafter (quadratic time effect: $B_{\text {movement }}=0.86$, $95 \%$ CI $[0.67,1.05], P<0.001 ; B_{\text {vistation }}=0.36,95 \%$ CI $[0.06,0.67]$, $P=0.019$; Fig. 5 and Supplementary Note 12). These analyses further confirm that the observed partisan gap in distancing increased over time.

Partisanship, physical distancing, and state policies. Possibly, local stay-at-home policies attenuate the observed partisan differences in physical distancing because these policies send out clear signals about the dangers of COVID-19. In line with this reasoning, 
Table 1 | Results of the main multi-level mixed-effects models in terms of reduction in general movement and visiting non-essential services

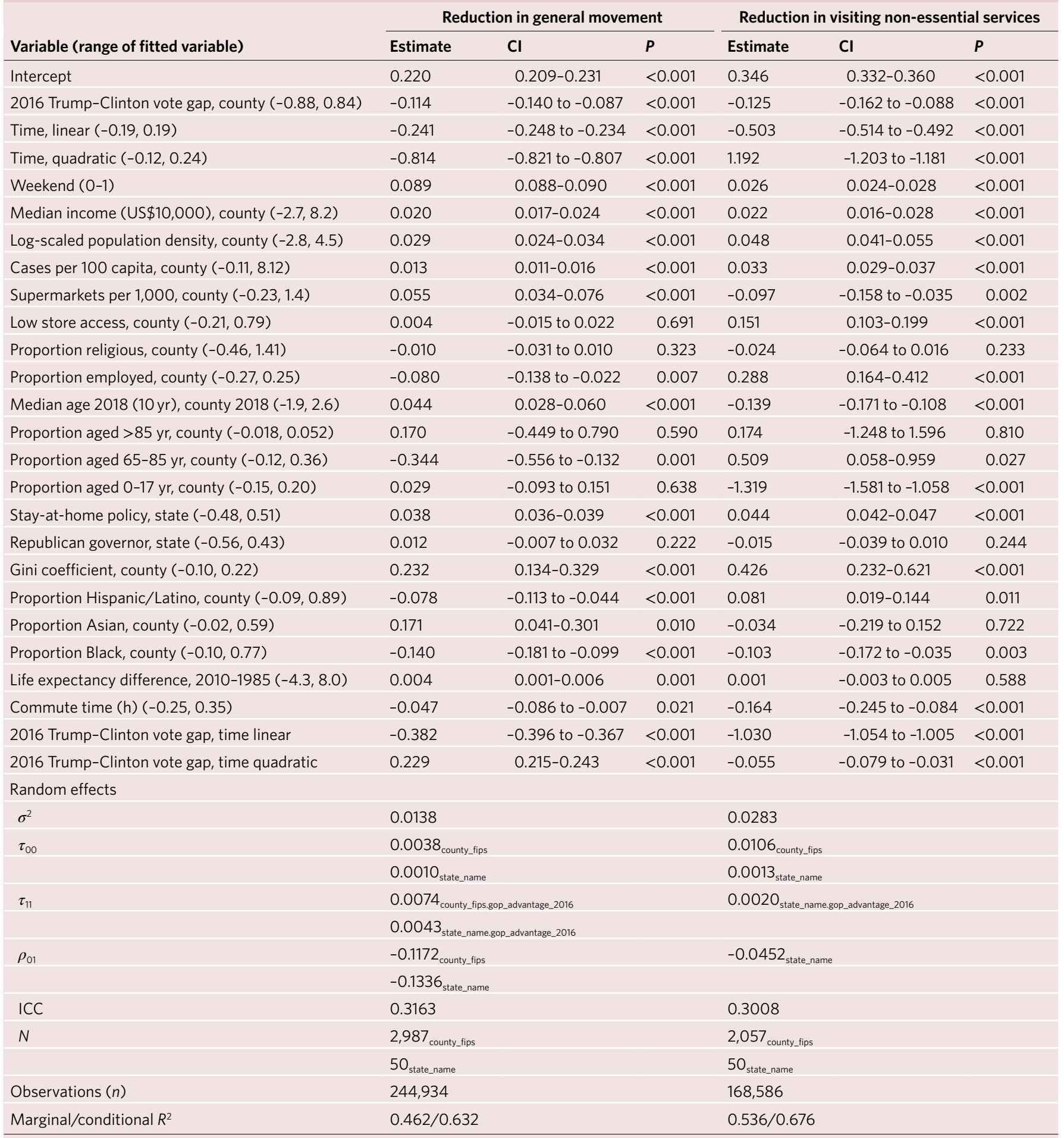

Given the scales of the physical distancing measures, an estimate of -0.114 , for instance, indicates an 11.4 percentage point smaller reduction in movement and visiting non-essential services as compared to before COVID-19. $\sigma^{2}$, within-group (residual) variance; $\tau_{00}$, between-group variance (variation between individual intercepts and average intercept); $\tau_{11}$, random slope variance (variation between individual slopes and average slope); $\tau_{01}$, random intercept slope covariance; $\rho_{01}$, random intercept slope correlation); ICC, intraclass correlation coefficient; GOP, Republican party; $N$, sample size.

we predicted that state-level stay-at-home orders would potentially reduce the observed partisan differences (see our preregistered hypotheses). Our data did not support this prediction. The link between voting for Trump and reduced physical distancing was actually stronger when stay-at-home orders were in effect, as evidenced by negative interactions between vote gap (with higher values indi- cating greater pro-Trump lean) and state policy: $B_{\text {movement }}=-0.017$, $95 \%$ CI $[-0.022,-0.013], P<0.001$ and $B_{\text {vistation }}=-0.009,95 \%$ CI $[-0.016,-0.001], P=0.030$ (examinations of detailed state policies, such as closing restaurants and closing schools, are included in Supplementary Tables 32 and 33 and Supplementary Note 13). These results align with recent work finding that Democratic coun- 


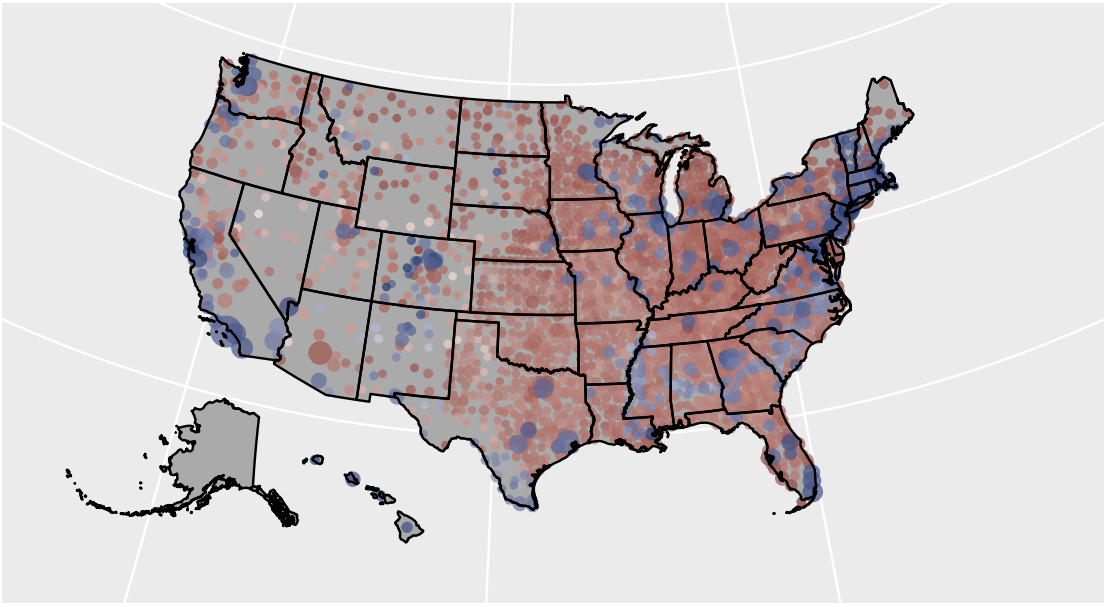

Percentage reduction

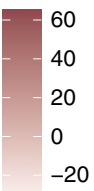

Percentage reduction

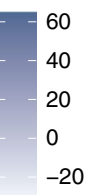

Fig. 2 | Map of predicted percent reduction in general movement as a function of county location and leaning pro-Trump versus pro-Clinton.

Predictions are derived from the multi-level models described in Table 1 and the main text. Each circle represents a county, its size proportional to its population. Lighter and darker colours indicate less or greater physical distancing, respectively. Blue indicates a Clinton-leaning county and red indicates a Trump-leaning county, based on 2016 voting figures.
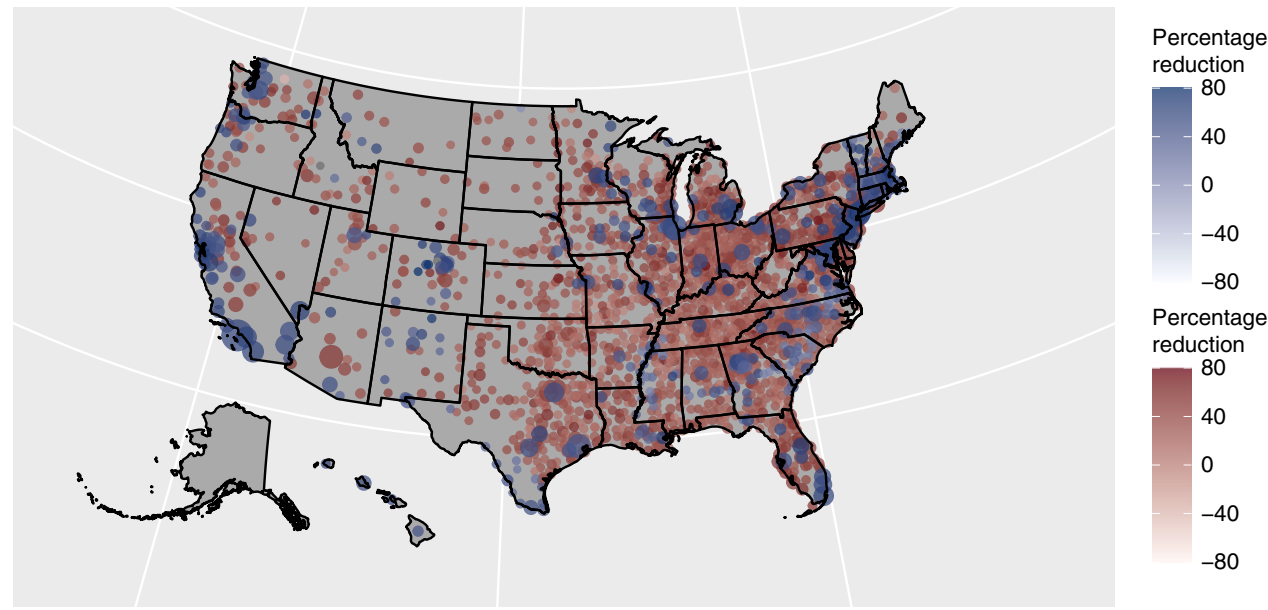

Fig. 3 | Map of predicted reduction in visiting non-essential services as a function of county location and leaning pro-Trump versus pro-Clinton. Predictions are derived from multi-level models described in Table 1 and the main text. Each circle represents a county, its size proportional to its population. Lighter and darker colours indicate less or greater physical distancing, respectively. Blue indicates a Clinton-leaning county and red indicates a Trump-leaning county, based on 2016 voting figures.

ties responded more quickly to stay-at-home orders than Republican counties at the start of the pandemic ${ }^{32}$.

We considered the possibility that variability in state stay-at-home policies explains our findings rather than county-level partisanship. For instance, stay-at-home orders may have been more stringent and remained in place longer in Democratic states or in states with Democratic governors, in turn leading Republican-leaning counties (which are more common in Republican states) to exhibit less physical distancing. Three supplementary analyses argued against this possibility, however. First, our results remained when adjusting for whether state policies were in effect on a specific date, state governors' political affiliation, and interactions between these variables and partisanship (Table 1 and Supplementary Table 31). Second, we also confirmed that our results remained when adding specific state policies (including closing restaurants, closing childcare, closing K-12 schools, closing non-essential business more generally, and closing religious institutions/gatherings), and the interactions between these policies and governor political party and partisanship, to our models (see Detailed State Policies section in the Supplementary Information and Supplementary Tables 32 and 33). And third, while there was some variation in our findings between states, most states were in line with the population average of the observed partisan differences (Supplementary Figs. 4-6 and Supplementary Note 14). In sum, though differences in state policies contribute to variation in county-level physical distancing, our results indicate that county-level partisanship nonetheless explains unique variance in physical distancing (see Supplementary Information for more details).

Partisan media and physical distancing. One method through which partisan viewpoints are propagated is partisan media. Potentially, then, watching more Republican- than Democratic-leaning media (for example, Fox News versus MSNBC) is linked to lower levels of physical distancing. Indeed, recent research has found a causal effect of Fox News viewership on reducing stay-at-home behaviour $^{33}$ and indicated that behavioural responses to COVID-19 

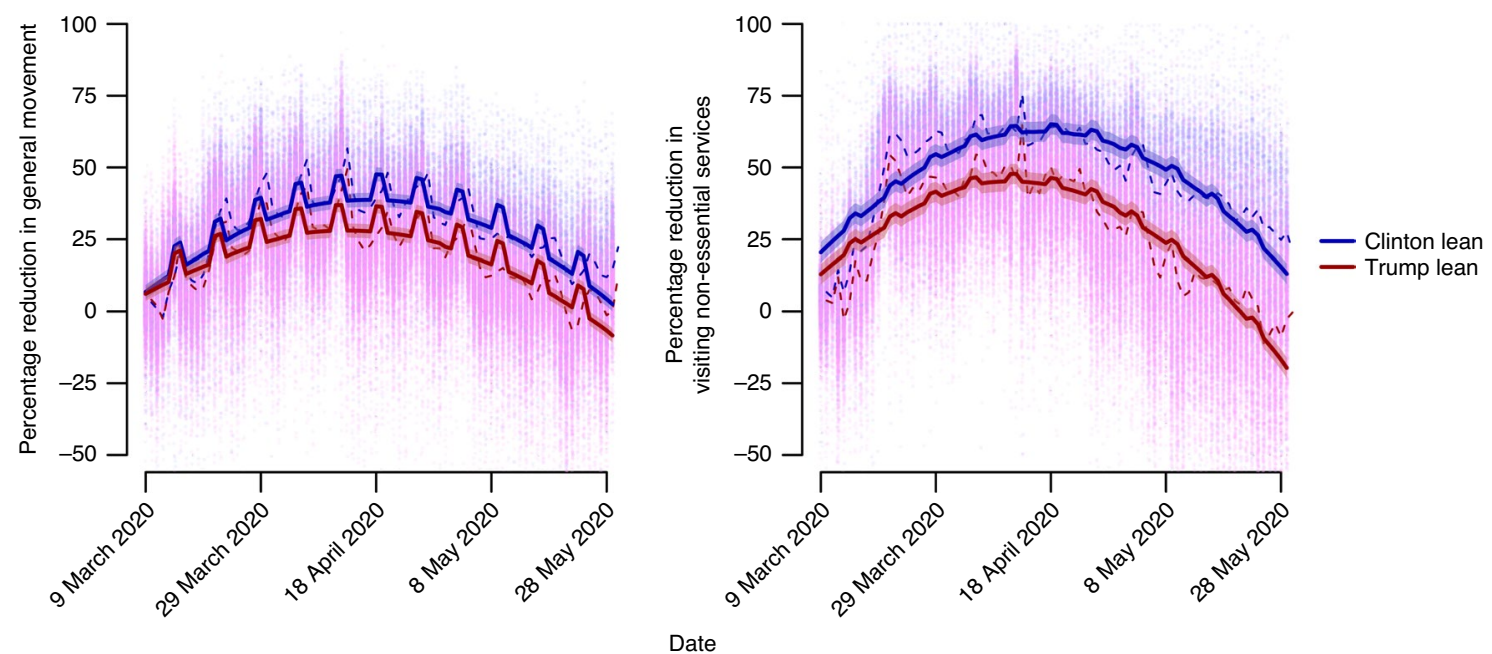

Fig. 4 | US counties' average physical distancing as a function of time and partisanship. Physical distancing was quantified as counties' percent reduction in general movement and visiting non-essential services as compared to before COVID 19 (before March 9). For graphing purposes, counties were binned as Trump lean if a greater percentage of residents voted for Trump in 2016, and as Clinton lean if a greater percentage of residents voted for Clinton. Each point represents one county (3,007 were included for general movement and 2,068 for non-essential services visitation across 82 days; the number of total data points was 246,574 and 169,480 , respectively). Dashed lines denote the daily average across counties, split by partisan lean. Bold blue and red lines represent the average predictions of vote gap from the multi-level models described in Table 1 and the main text. Error bands are bootstrapped $95 \%$ prediction intervals.
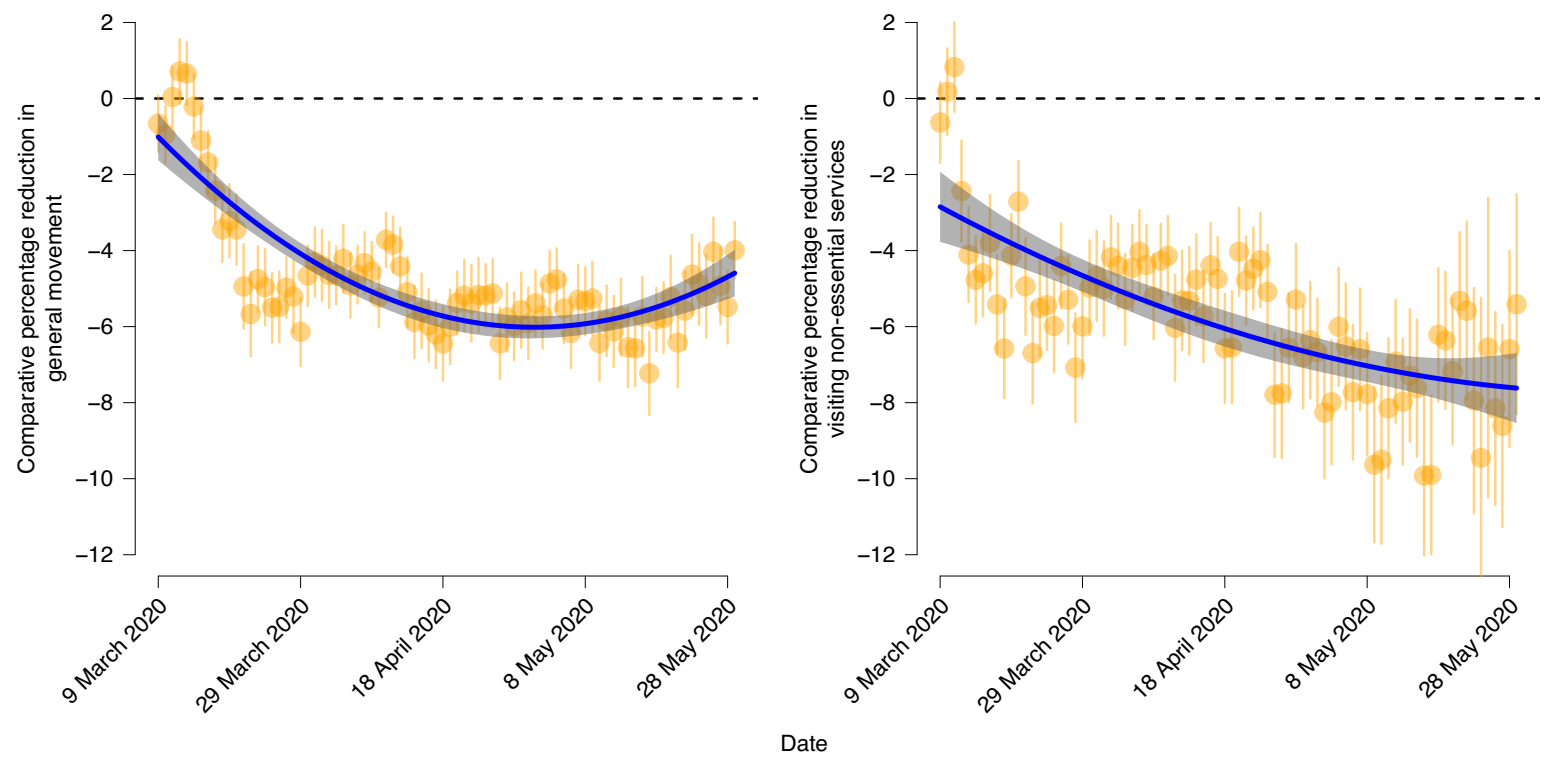

Fig. 5 | Percentage reduction in general movement and visiting non-essential services (relative to pre-COVID, before 9 March) for Trump as compared to Clinton voting counties (negative values indicate less physical distancing in Trump counties) as a function of time (between 9 March and 29 May 2020). Individual points represent regression coefficients. More negative coefficients represent a greater partisan gap. For instance, -6 on the yaxis indicates 6 percentage points less physical distancing in Trump counties (+1s.d. pro-Trump vote lean) on a specific day. Given the date range of 82 days, 82 observations are included for each of the two physical distancing measures. Blue lines represent predictions from linear regression models with linear and quadratic time terms. Generalized additive models models of these data can be found in the Supplementary Information. Error bands, mean \pm 2 s.e.

change in line with the specific cable news show that people watch (for example, Tucker Carlson Tonight versus Hannity) ${ }^{34}$. To examine whether viewing Republican- versus Democratic-leaning media is associated with differences in physical distancing, we analysed viewership data from SimplyAnalytics. We found that the degree to which people in different counties watched Fox News over CNN and MSNBC was associated with lower levels of physical distancing
$\left(B_{\text {movement }}=-0.053,95 \%\right.$ CI $[-0.076,-0.030]$ and $B_{\text {visitation }}=-0.099$, 95\% CI [-0.136, -0.062$], P<0.001$; Supplementary Fig. 2 and Supplementary Note 15). Importantly, these results were found while accounting for all covariates (Main Model in Supplementary Tables 27 and 28) and remained when additionally controlling for interactions between watching Fox News (over CNN and MSNBC) and these covariates: $P<0.001$ (Saturated Model in Supplementary 
Tables 27 and 28). And, as was the case for partisanship as assessed by 2016 vote gap, the observed partisan media differences in physical distancing strengthened over time (Supplementary Tables 27, 28, and 30 and Supplementary Figs. 2 and 3).

We also tested whether consuming Republican-leaning media and 2016 pro-Trump versus pro-Clinton voting account for independent variance in physical distancing. Supporting this possibility, we found that Fox News consumption (over MSNBC and CNN) still negatively related to physical distancing when including 2016 vote gap, the interactions between vote gap and linear and quadratic time, and the interaction between vote gap and Fox News lean in the models: $B_{\text {visitation }}=-0.038,95 \%$ CI $[-0.063,-0.014]$, $P=0.002$ and $B_{\text {visitation }}=-0.053,95 \%$ CI $[-0.092,-0.013], P=0.009$ (Supplementary Table 29). Furthermore, in these analyses the coefficients for partisan vote gap and Fox News lean both decreased (Table 1 and Supplementary Tables 27 and 29), suggesting that Republican-leaning media consumption may in part account for the observed covariance between vote gap and physical distancing (and vice versa).

Linking physical distancing to COVID-19 infections and fatalities. We next examined whether the observed partisan differences in distancing relate to COVID-19 infections and fatalities. Applying three-level, mixed-effects models, with counties nested within states, we first tested whether counties' time-lagged physical distancing is associated with decreased COVID-19 infection and fatality growth rate. Growth rates were examined because the main goal of physical distancing is to 'flatten the curve', or the spread, of coronavirus disease (see Methods for calculation) ${ }^{34,35}$.

Given the time lag between contracting COVID-19, experiencing symptoms, and receiving a positive test result, physical distancing should not have an immediate impact on infection or fatality growth rate. In line with this reasoning, we considered the length of the delay between physical distancing and subsequent reductions in infections and fatalities. We treated the incubation period of COVID-19 as the time from exposure to symptom onset ${ }^{36-39}$. Although this period may include a period of asymptomatic viral shedding, we did not account for this in our analysis ${ }^{39}$. Based on previous work, we estimated the incubation period of COVID-19 to be between 2 and 11 days (median $4-5$ days) ${ }^{36-39}$. We then estimated that receiving positive test results typically takes at least 1 day (and often takes much longer). Given these estimates, physical distancing could begin to reduce counties' infection growth rate as early as 3 days thereafter, but more likely begins to have a measurable impact around 7-8 days later ( $\sim$-day incubation period plus $\sim 3$ days to seek and receive test results). Importantly, this estimate does not represent when the impact of physical distancing is strongest. That is, physical distancing should relate to decreased infection growth rate more and more strongly as delayed testing is completed, residual infections die out, and cases with long incubation periods pass, until eventually this link peaks and then starts to decline (Supplementary Figs. 12-15) ${ }^{40}$.

We conducted a series of mixed-effects models to identify the lag time in which physical distancing most strongly relates to reduced infections. Specifically, we examined the predictive power of rolling 7 -day averages of general movement and visiting non-essential services $\left(\left(p_{t}+p_{t-1}+\ldots+p_{t-6}\right) / 7 ; t=\{35,34, \ldots, 7\}\right)$, where $t$ is lag day and $p$ is physical distancing score) on infection growth rate. Both for general movement and visiting non-essential services, physical distancing between 17 and 23 days before a given date ( $z$-scored, 7 -day lagged average) most strongly related to lower infection growth rate on that date: $B_{\text {movement }}=-3.05,95 \%$ CI $[-3.15,-2.94]$ and $B_{\text {visitation }}=-3.53,95 \%$ CI $[-3.65,-3.41], P<0.001$ (Table 2 and Supplementary Figs. 12 and 13; the first 7-day lag to negatively relate to infections was between 6 and 12 days for general movement and 7 and 13 days for visitation). In other words, a reduction in infection growth rate on a specific day was best accounted for by the degree to which people in a county were physically distancing between 17 and 23 days earlier (Supplementary Information).

Importantly, this link remained when including all covariates from our earlier analyses (for example, population density, distance travelling to work, age; see Methods) and when additionally including non-time-lagged physical distancing (physical distancing on the same day) in these models: $B_{\text {movement }}=-3.31,95 \%$ CI $[-3.42,-3.21]$ and $B_{\text {visitation }}=-4.54,95 \%$ CI $[-4.66,-4.41], P<0.001$ (Table 2). Moreover, supporting the unique relationship between time-lagged physical distancing and subsequent infections, counties' non-lagged physical distancing (that is, same-day physical distancing) was associated with a higher rather than lower infection growth rate: $B_{\text {movement }}=1.66,95 \%$ CI $[1.55,1.77]$ and $B_{\text {visitation }}=2.94,95 \%$ CI $[2.81$, 3.08], $P<0.001$ (Table 2). These positive same-day relationships between distancing and growth rate likely reflect that counties hit hardest by the pandemic were also those that responded by engaging most in physical distancing (see Supplementary Information for cross-lagged analyses substantiating this claim).

Turning to fatalities, estimates of the time lag from COVID-19 infection to death vary between 2 and 4 weeks (approximately 1-2 weeks after symptoms appear) ${ }^{41,42}$. Using the same 7-day lagged approach used for infections, we found that counties' physical distancing 25-31 days before a given day most strongly related to lower fatality growth on that day: $B_{\text {movement }}=-0.67,95 \% \mathrm{CI}[-0.74,-0.59]$ and $B_{\text {visitation }}=-0.69,95 \%$ CI $[-0.79,-0.59], P<0.001$ (Table 2 and Supplementary Figs. 14 and 15). As was true for infections, these results remained when including all covariates and when including non-lagged physical distancing in the models: $B_{\text {movement }}=-0.70$, $95 \%$ CI $[-0.78,-0.62]$ and $B_{\text {visitation }}=-0.81,95 \%$ CI $[-0.91,-0.70]$, $P<0.001$ (Table 2). And, as was also true for infections, non-lagged distancing was associated with higher rather than lower fatalities: $B_{\text {movement }}=0.45,95 \% \mathrm{CI}[0.38,0.51]$ and $B_{\text {visitation }}=0.55,95 \% \mathrm{CI}$ $[0.46,0.64], P<0.001$ (Table 2). Collectively, these results suggest that counties' degree of physical distancing 17-23 and 25-31 days earlier best accounts for reductions in infection and fatality growth rates, respectively.

Linking partisanship and physical distancing to COVID-19 infections and fatalities. Finally, we tested whether partisan-linked failures to physically distance during the pandemic related to higher infection and fatality growth rates. We approached this analysis by testing mediation models in Stata following multi-level mediation procedures $^{43}$. These mediation models included three-level, mixed-effects models as in the analyses reported throughout this article (Supplementary Note 16). The models included counties' pro-Trump voting ( $z$-scored) as the predictor variable, lagged physical distancing (general movement and visiting non-essential services lagged 17-23 days for infections and 25-31 days for fatalities; $z$-scored) as the mediators, infection growth rate or fatality growth rate as the outcome variables and all covariates as control variables (see Supplementary Information for more detail).

The mediation analyses revealed significant indirect links between counties' pro-Trump voting and infection growth rate via physical distancing (lagged 17-23 days): $B_{\text {movement }}=0.855,95 \%$ CI $[0.622$, $1.088]$ and $B_{\text {visitation }}=0.896,95 \%$ CI $[0.679,1.113], P<0.001$ (Table 3 ). Regarding total effects, Trump-leaning counties on average exhibited marginally higher infection growth rates than Clinton-leaning ones between 26 March and 29 May 2020: $B_{\text {movement }}=0.272,95 \% \mathrm{CI}$ $[-0.020,0.564]$ and $B_{\text {visitation }}=0.313,95 \%$ CI $[0.032,0.594], P<0.068$ (Total Effects in Table 3 and Supplementary Note 17); however, this would not have been the case if Trump-leaning counties had physically distanced to the same degree as more Clinton-leaning counties. If they had done so, Trump-leaning counties would actually have had lower infection growth rates than Clinton-leaning ones 
Table 2 | Results of multi-level, mixed-effects models testing whether physical distancing is linked to infection and fatality growth rates

\begin{tabular}{|c|c|c|c|c|c|}
\hline & Estimate & s.e. & $z$ & $\mathrm{Cl}$ & $P$ \\
\hline \multicolumn{6}{|l|}{ Infection growth rate } \\
\hline \multicolumn{6}{|l|}{ General movement } \\
\hline 17-23 days lagged physical distancing (absent covariates) & -3.05 & 0.05 & -56.29 & -3.15 to -2.94 & $<0.001$ \\
\hline 17-23 days lagged physical distancing (all covariates included) & -3.24 & 0.05 & -59.30 & -3.34 to -3.13 & $<0.001$ \\
\hline $\begin{array}{l}\text { 17-23 days lagged physical distancing (all covariates and non-lagged physical distancing } \\
\text { included) }\end{array}$ & -3.31 & 0.05 & -61.95 & -3.42 to -3.21 & $<0.001$ \\
\hline Non-lagged physical distancing (all covariates included) & 1.66 & 0.06 & 30.02 & $1.55-1.77$ & $<0.001$ \\
\hline \multicolumn{6}{|l|}{ Visiting non-essential services } \\
\hline 17-23 days lagged physical distancing (absent covariates) & -3.53 & 0.06 & -57.41 & -3.65 to -3.41 & $<0.001$ \\
\hline 17-23 days lagged physical distancing (all covariates included) & -4.24 & 0.06 & -65.33 & -4.37 to -4.11 & $<0.001$ \\
\hline $\begin{array}{l}\text { 17-23 days lagged physical distancing (all covariates and non-lagged physical distancing } \\
\text { included) }\end{array}$ & -4.54 & 0.06 & -71.51 & -4.66 to -4.41 & $<0.001$ \\
\hline Non-lagged physical distancing (all covariates included) & 2.94 & 0.07 & 42.14 & $2.81-3.08$ & $<0.001$ \\
\hline \multicolumn{6}{|l|}{ Fatality growth rate } \\
\hline \multicolumn{6}{|l|}{ General movement } \\
\hline 25-31 days lagged physical distancing (absent covariates) & -0.67 & 0.04 & -17.65 & -0.74 to -0.59 & $<0.001$ \\
\hline 25-31 days lagged physical distancing (all covariates included) & -0.71 & 0.04 & -17.84 & -0.79 to -0.64 & $<0.001$ \\
\hline $\begin{array}{l}25-31 \text { days lagged physical distancing (all covariates and non-lagged physical distancing } \\
\text { included) }\end{array}$ & -0.70 & 0.04 & -17.59 & -0.78 to -0.62 & $<0.001$ \\
\hline Non-lagged physical distancing (all covariates included) & 0.45 & 0.03 & 13.73 & $0.38-0.51$ & $<0.001$ \\
\hline \multicolumn{6}{|l|}{ Visiting non-essential services } \\
\hline 25-31 days lagged physical distancing (absent covariates) & -0.69 & 0.05 & -13.37 & -0.79 to -0.59 & $<0.001$ \\
\hline 25-31 days lagged physical distancing (all covariates included) & -0.82 & 0.05 & -15.12 & -0.93 to -0.72 & $<0.001$ \\
\hline $\begin{array}{l}25-31 \text { days lagged physical distancing (all covariates and non-lagged physical distancing } \\
\text { included) }\end{array}$ & -0.81 & 0.05 & -14.80 & -0.91 to -0.70 & $<0.001$ \\
\hline Non-lagged physical distancing (all covariates included) & 0.55 & 0.05 & 12.15 & $0.46-0.64$ & $<0.001$ \\
\hline
\end{tabular}

Physical distancing 17-23 days before a given date related to decreased infection growth rates on that date. Physical distancing 25-31 days before a given date related to decreased fatality growth rates on that date.

between 26 March and 29 May 2020: $B_{\text {movement }}=-0.583,95 \%$ CI $[-0.915,-0.251]$ and $B_{\text {visitation }}=-0.582,95 \%$ CI $[-0.912,-0.253]$, $P<0.002$ (Direct Effects in Table 3).

Similar indirect effects were also observed for fatality growth rate: $B_{\text {movement }}=0.183,95 \% \mathrm{CI}[0.090,0.277]$ and $B_{\text {visitation }}=0.176$, $95 \%$ CI [0.087, 0.264], $P<0.001$ (lagged 25-31 days earlier; Table 3). Regarding total effects, Trump-leaning counties exhibited lower fatality growth rates than Clinton-leaning ones: $B_{\text {movement }}=-0.417$, $95 \%$ CI $[-0.647,-0.188]$ and $B_{\text {visitation }}=-0.533,95 \%$ CI $[-0.850$, $-0.216], \quad P<0.001$ (between 3 April and 29May 2020; see Total Effects in Table 3); however, these counties would have exhibited even lower fatality growth rates had they physically distanced to the same extent as more Clinton-leaning counties: $B_{\text {movement }}=-0.601,95 \%$ CI $[-0.858,-0.344]$ and $B_{\text {visitation }}=-0.709$, $95 \%$ CI $[-1.050,-0.368], P<0.001$ (see Direct Effects in Table 3 and Supplementary Note 18).

This pattern of results was robust. The observed mediations remained consistent when including employment types as further control variables $(P<0.003$; Supplementary Table 34$)$; when testing earlier date ranges for the lagged mediators (for example, $26 \mathrm{March}$ to 29 May for infections), $P<0.005$ (Supplementary Table 35); when excluding all cases in which growth rate was 0 (to account for potential zero inflation in the data), $P<0.001$ (Supplementary Table 36); when calculating infection and fatality growth rates using moving averages, $P<0.001$ (Supplementary Table 37); and when including specific state policies (for example, closing restaurants, closing schools) as additional control variables, $P<0.001$ (Supplementary Table 38).

To put our findings into context, our model indicated that extremely pro-Trump-voting counties $(+2 z$-score in the vote gap variable) experienced a daily infection growth rate of 0.59 percentage points higher than average (between 26 March and 29 May 2020, the average daily infection growth rate across all counties in this date range was 5.72 percentage points; Supplementary Note 19). Such pro-Trump counties would have experienced a daily infection growth rate of 1.17 percentage points less on average, however, if they had socially distanced to the same extent as the average county-a swing of 1.76 percentage points in infection growth rate. Our findings thus suggest that partisan differences in physical distancing were linked to higher growth rates of infections and fatalities in pro-Trump counties than necessary.

Finally, we reconducted our mediation analyses when calculating infection and fatality growth rate using an exponential model of disease spread (Supplementary Information; growth rate $r=\ln \left(I_{t+1} / I_{t}\right)$, where $I$ is the cumulative number of infections and $t$ is the time (day) (ref. $\left.{ }^{35}\right)$ ). Applying an exponential growth rate is illuminating from an epidemiological perspective, because COVID-19 would initially spread exponentially in the scenario of a disease spreading within a closed, homogeneous population without interventions $s^{29,35,44,45}$. Importantly, when doing so, we observed the same mediation results as above: $P<0.001$ (Supplementary Table 39). 
Table 3 | Output of mediation models

\begin{tabular}{|c|c|c|c|c|c|}
\hline & Predictor variable & \multicolumn{3}{|c|}{ Mediator } & Outcome variable \\
\hline Mediation 1 & Partisanship & \multicolumn{3}{|c|}{ Lagged reduction in general movement (17-23 days previously) } & Infection growth rate \\
\hline Total effect & $B=0.272$ & s.e. $=0.149$ & $z=1.83$ & $P=0.067$ & $95 \% \mathrm{Cl}[-0.020,0.564]$ \\
\hline Indirect effect & $B=0.855$ & s.e. $=0.119$ & $z=7.19$ & $P<0.001$ & $95 \% \mathrm{Cl}[0.622,1.088]$ \\
\hline Mediation 2 & Partisanship & \multicolumn{3}{|c|}{ Lagged reduction in visiting non-essential services (17-23 days previously) } & Infection growth rate \\
\hline Total effect & $B=0.313$ & s.e. $=0.143$ & $z=2.19$ & $P=0.029$ & $95 \% \mathrm{Cl}[0.032,0.594]$ \\
\hline Indirect effect & $B=0.896$ & s.e. $=0.111$ & $z=8.09$ & $P<0.001$ & $95 \% \mathrm{Cl}[0.679,1.113]$ \\
\hline Mediation 3 & Partisanship & \multicolumn{3}{|c|}{ Lagged reduction in general movement (25-31 days previously) } & Fatality growth rate \\
\hline Total effect & $B=-0.417$ & s.e. $=0.117$ & $z=-3.56$ & $P<0.001$ & $95 \% \mathrm{Cl}[-0.647,-0.188]$ \\
\hline Direct effect & $B=-0.601$ & s.e. $=0.131$ & $z=-4.58$ & $P<0.001$ & $95 \% \mathrm{Cl}[-0.858,-0.344]$ \\
\hline Indirect effect & $B=0.183$ & s.e. $=0.048$ & $z=3.85$ & $P<0.001$ & $95 \% \mathrm{Cl}[0.090,0.277]$ \\
\hline Indirect effect & $B=0.176$ & s.e. $=0.045$ & $z=3.89$ & $P<0.001$ & $95 \% \mathrm{Cl}[0.087,0.264]$ \\
\hline
\end{tabular}

The link between partisanship (vote share for Trump over Clinton in 2016, z-scored) and COVID-19 infection and fatality growth rate was mediated by time-lagged physical distancing (reduction in general movement and visiting non-essential services $17-23$ and 25-31 days earlier, respectively; $z$-scored).

It is possible that the estimated indirect effects are skewed by differences in reporting or tracking infections (for example, certain areas testing more than others). To begin to account for this possibility, we examined and found that the observed indirect effects of county partisanship on infection growth rate remained when individually testing these mediations within states and then aggregating these results via meta-analysis: $B=0.417, z=3.43, P<0.001$ (Supplementary Information). These analyses help address reporting concerns because, although reporting differences may exist across states, it is less likely that they exist within states (the utilized mixed-effects modelling should also help allay this concern; Supplementary Note 20). Nonetheless, biased estimates in reporting and testing remain possible, if not probable, and thus are a limitation of our results.

\section{Discussion}

The present work used the geotracking data of approximately 15 million people per day across the United States to examine whether partisan identity is linked to objective measures of physical distancing during the coronavirus pandemic. We found that Republican-leaning counties exhibited lower physical distancing than Democratic-leaning counties, both in terms of reducing their overall movement and reducing their visiting of non-essential services (e.g., restaurants, clothing stores). Counties that voted for Trump in the 2016 election exhibited a 24\% drop in general movement and visiting non-essential services between 9 March and 29 May 2020, while counties that voted for Clinton exhibited a $\sim 38 \%$ drop (a 14\% difference in physical distancing). Moreover, this partisan gap remained consistent after adjusting for numerous third variables, including counties' number of COVID-19 cases per capita, population density, median income, wealth distribution, travel time to work, and racial and age make-up, among other factors. These data suggest that partisan differences in self-reported attitudes toward COVID-19 (refs. ${ }^{18-22}$ ) are mirrored by behavioural differences at the US county level.

We also linked the observed partisan differences in physical distancing to COVID-19 infections and fatalities. Mediation analyses revealed that reduced physical distancing in counties was linked to a subsequent increase in COVID-19 infection and fatality growth rates roughly $17-23$ and $25-31$ days later, respectively. These results imply that Trump-leaning counties could have curbed their infection and fatality growth rates if they had distanced to the same degree as Clinton-leaning counties did. Such findings provide evidence that partisanship in the United States during the COVID-19 pandemic may have had meaningful-and severe-health-related consequences. Partisanship may therefore be an important risk factor during a public health crisis, one that is probably especially relevant when the electorate is highly polarized and leaders fail to generate bipartisan support for public health measures ${ }^{17}$.

The observed partisan differences appear to be relatively strong as well as highly robust. Partisanship was more strongly associated with physical distancing than most of the covariates included in our models, including more traditionally examined health-related variables such as economic and social indicators. The relationship between partisanship and distancing also held after statistically adjusting for numerous control variables, and for interactions between partisanship and these control variables. The robustness of these findings is consistent with findings from independent research groups who have also observed links between partisanship and physical distancing using different data sources ${ }^{46,47}$. Taken together, these findings provide a more complete picture of partisanship during a pandemic and establish it as an important risk factor for COVID-19 in the United States.

The observed partisan gap in physical distancing was not limited to the beginning of the COVID-19 pandemic. Although we had expected that this difference would attenuate as the pandemic worsened after 23 March 2020 (Supplementary Note 21), we instead found that the partisan gap actually increased over time (Fig. 5). We also expected that intervention by local government would attenuate the observed partisan differences. Again, instead we found that stay-at-home orders actually exacerbated the observed partisan gap; that is, stay-at-home orders appeared to be more successful in encouraging Democratic counties to physically distance than Republican ones. This difference may be driven by more Republican-leaning counties ignoring local stay-at-home orders in light of national messaging from right-wing media and federal leaders; or, Republicans may simply hold a greater distrust of government than Democrats ${ }^{48}$. These issues require further investigation 
to determine how to intervene locally to ensure greater compliance with public health recommendations.

The observed partisan differences may be partially driven by Republican counties experiencing fewer COVID-19 infections and fatalities than Democratic ones at the start of the pandemic. Simply put, for Republicans, the threat of COVID-19 may have been less salient. Arguing against this possibility, our findings remained when adjusting for counties' infections per capita (Table 1); doing so should account for Republican counties being objectively less threatened during the examined time frame (between 9 March and 29 May 2020). Moreover, desired political outcomes (for example, wanting the pandemic to be less serious than it is for political reasons), and not one's actual experiences with COVID-19 (for example, knowing someone who was infected), appear to drive self-reported partisan differences in concern about the pandemic ${ }^{49}$. As such, it seems that objective risks of infection do not account for the observed partisan differences in physical distancing.

In our view, a more likely, albeit partial, explanation for the observed results is media polarization or a 'broadcasting effect' ${ }^{15,50}$. Republican-leaning media outlets appear to have downplayed the dangers of the coronavirus as compared to more Democratic outlets. For instance, in early March, Fox News repeatedly claimed that the coronavirus was less dangerous than influenza and even referred to other media reports as a hoax (although some commentators took it more seriously than others, and the organization took the pandemic more seriously by mid-March 2020) ${ }^{51}$. Consistent with this messaging about COVID-19, we found that US counties that consumed more Fox News than Democratic-leaning outlets (MSNBC and $\mathrm{CNN}$ ) exhibited less physical distancing. These findings also remained significant when controlling for counties' partisanship in terms of 2016 voting. These results, along with self-report data indicating similar findings $\mathrm{s}^{22}$ and evidence indicating a causal link between Fox News viewership and decreased social distancing ${ }^{33}$, suggest that Republican-leaning media downplaying the virus at the start of the pandemic may have signalled to Republicans that they should not take the virus very seriously, in turn potentially in part causing the observed partisan differences. This possibility underscores the importance of considering communication and mass media when designing public health messaging.

Of course, other factors may help account for the observed differences as well. For instance, political role models may have signalled different actions as appropriate responses to the pandemic (for example, not wearing versus wearing a mask). As an example, President Donald Trump referred to criticisms about his administration's response to the pandemic as 'their new hoax'. Moreover, public events hosted by Donald Trump in Tulsa and Arizona (June 2020) revealed, accordingly, that at least some of his supporters were willing to attend high-risk events such as large, indoor gatherings.

Finally, we consider that conservative versus liberal political ideologies may lead individuals to respond differently to threat or specifically to the threat of a viral pandemic ${ }^{52,53}$. We find this conclusion unlikely, however, given that conservatives were actually more likely than liberals to report being worried about the potential spread of a different virus-Ebola-in the United States in 2014 (ref. ${ }^{54}$ ). As such, it seems that our findings are more likely to be driven by modelling the behaviour of political role models and political identity (that is, political group loyalty) than by political ideology (that is, political values) $)^{7,16,55,56}$. Indeed, Democrats are not only more likely than Republicans to say they prioritize stay-at-home orders, but this partisan difference is most pronounced among highly identified group members ${ }^{57}$.

Our findings draw from a very large sample of real behaviour and are robust to several model specifications, but nevertheless have limitations. First, these data are correlational and cannot be used to draw causal conclusions. Second, the data are county-level aggregates and thus do not tell us whether individual Republicans engage in less physical distancing than Democrats (although self-reported data indicate they are likely to) ${ }^{19}$. Likewise, although we accounted for numerous structural state-level factors (for example, governor's political party, types of state policy), it remains plausible that certain state-level factors explain a portion of the observed findings. Third, the current results are limited to the United States; it remains unclear whether partisanship or political polarization played a role in COVID-19 responses in other countries. Fourth, we did not consider the role of face masks or other public health behaviours in the present findings. That is, our findings may or may not extend to other preventative COVID-19 practices and, additionally, these other practices may covary with distance and therefore help account for subsequent infections and mortality. Fifth, infection and fatality counts may be unreliable. For instance, certain areas may have failed to provide proper testing or failed to count deaths at home as COVID-19 fatalities. As such, our mediation results should be approached cautiously. Sixth, several of the variables we examined failed to capture nuanced variance; for instance, although we controlled for counties' average number of supermarkets, these values may vary substantially within a county (creating 'food deserts' that we do not account for). And, although we control for counties' type of employment in our analyses, physical distancing may vary greatly within these employment types. Finally, people in pro-Trump counties may need to travel further to shop for essential goods or for work. However, we found consistent results when statistically adjusting for access to supermarkets and average time to travel to work. Nevertheless, future research should examine individual behavioural differences using causal tests to help determine whether partisanship is causally related to public health behaviour.

Although few things bind groups together like a common obstacle, the COVID-19 pandemic has had a deeply partisan flavour in the United States (and seemingly continues to do so). Here we found that partisanship is associated with differences in physical distancing behaviour at the level of US counties-and that this degree of physical distancing may have impacted counties' subsequent COVID-19 infection and fatality growth rates. This suggests that partisanship might be an important risk factor for the current pandemic and, potentially, for other public health crises. We encourage researchers who study other health issues to consider the role that partisanship might play in health-related decision making. Our work highlights the possibility that political partisanship in the United States is now sufficiently far-reaching and pernicious enough to threaten the health of citizens during a pandemic.

\section{Methods}

Our research complies with all relevant ethical regulations. Because the data in question include aggregated county-level data collected by an external source (Unacast), these are categorized as exempt according to the Yale University Institutional Review Board. We are unaware of whether participants were compensated. Participants completed opt-in consent. The sample included approximately 15 million smartphone users per day in the United States. Given the aggregated nature of these data, the sex, age and exact number of participants are unknown. No statistical methods were used to predetermine sample size (in terms of the number of included US counties) but our sample was quite large ( 3,025 counties were included in our analyses). This sample size provides sufficient statistical power to observe relationships of even a very small size. Preregistrations of hypotheses were made, see above. These preregistrations specify two hypotheses - that the observed partisan differences would reduce with time and would reduce when stay-at-home orders were in effect. These preregistrations do not speak to any other hypotheses and do not include clear analysis plans or model specifications, and thus solely qualify as broad preregistrations of these two specific hypotheses (Supplementary Notes 9 and 10).

Physical distancing. We examined people's physical distancing behaviours in 3,025 US counties (those with a population of at least 2,000; Supplementary Note 22) between 9 March and 29 May 2020. Physical distancing was assessed via a county's percentage reduction in general movement and visiting non-essential services (for example, barbers, restaurants, clothing stores; Supplementary Note 23) relative to the period before COVID-19 struck the United States with force (before 9 March; Supplementary Note 24). Physical distancing on a specific 
day was calculated as the change in a county's general movement and visiting non-essential services from the same day of the week during the 4 weeks leading up to 9 March (percentage reduction in general movement: $M=0.21$, s.d. $=0.19$; percentage reduction in general movement: $M=0.31$, s.d. $=0.30$ ). For example, a county's level of physical distancing on Monday, 9 March was calculated as the percentage reduction in movement and non-essential visits from the average levels of movement and non-essential visits on the four preceding pre-COVID Mondays (10, 17 and 24 February and 2 March) in that county. In our main analyses, we included 3,007 counties across 82 days when examining general movement ( 18 counties were excluded on account of missing partisanship data; see below), and 2,068 counties across 82 days when examining non-essential visitation (957 counties were excluded, and some additional physical distancing data points, for missing partisanship or missing visitation data). This resulted in the inclusion of a total of 248,050 and 169,480 physical distancing data points when examining general movement and non-essential visitation, respectively.

The two physical distancing variables assessed-general movement and visiting non-essential services-were calculated by Unacast (a software company that provides location tracking and map services) using approximately 15 million smartphone GPS coordinates across the United States per day. Unacast gathers GPS data from thousands of opt-in consent apps. These GPS data are then categorized in terms of stops (a device being stationary over a period of time) and travel events (a device moving). General movement is thus, for instance, calculated as the total time travelled for each device in a county, averaged across all the devices in that county (Supplementary Information provides greater detail on how the physical distancing variables were constructed). The data were anonymized, in that GPS coordinates were aggregated at the county level (the locations of specific individuals were not shared with the authors)

Partisanship and the vote gap. A continuous index of partisanship was created using the county-level voting gap in the 2016 election-that is, the proportion of total votes for Donald Trump minus the proportion of total votes for Hillary Clinton. Average vote share across the 3,007 counties for which we obtained data was $31 \%$ greater for Trump: $M=0.31$, s.d. $=0.30$, minimum $=-0.89$, maximum $=0.85$. Binning counties according to Trump versus Clinton lean produced 480 Clinton counties (average vote share favouring Clinton: $M=-0.23$, s.d. $=0.19$ ) and 2,527 Trump counties (average vote share favouring Trump: $M=0.41$, s.d. =0.19). Greater detail of all the included measures can be found in the Supplementary Information and on our OSF project page (https://osf.io/ u5pmw/?view_only=33f0691a7e694276bef606 cb3e22d141).

Fox News preference. We created a Fox News preference score by subtracting the proportion of people watching CNN and MSNBC (averaged) from the proportion of people watching Fox News per US county. Across counties, Fox News was on average watched $7.6 \%$ more (in terms of proportion of population) than $\mathrm{CNN}$ and MSNBC: $M=0.076(7.6 \%)$, s.d. $=0.004$, minimum $=0.059$, aximum $=0.098$.

Control variables. All covariates were, insofar as possible, kept on their original scale to aid understanding of regression coefficients reported in the Supplementary tables. The covariates included weekend $(0=$ weekday, $1=$ weekend); COVID19 infections per 100 capita (on each date for the included date range, mean centred); state policy $(0=$ stay-at-home order not in effect and $1=$ stay-at-home order in effect on each date for the included date range, mean centred); party affiliation of state governor $(0=$ Republican, $1=$ Democrat, mean centred $)$; population density (log, standardized); median household income in 2018 (in units of US\$10,000, mean centred; degree of income inequality (Gini coefficient, mean centred); median age in 2018 (mean centred); proportion of population aged $0-17$ years (mean centred); proportion of population aged $>65$ years (mean centred); proportion of population aged $>85$ years (mean centred); race/ethnicity (proportion of population Black, Hispanic and Asian, all mean centred); low access to grocery stores (for example, because of distance or income, mean centred); number of grocery stores per 1,000 people (mean centred); mean travel time to work (hours, mean centred); religiosity (mean centred); change in life expectancy from 1985 to 2010 (years, mean centred); and proportion employed (mean centred). We also controlled counties' percentage of employment in various types of profession (for example, agriculture, finance, manufacturing) in additional analyses (Supplementary Table 24). More details regarding these control variables, descriptive statistics, and the strength of links between these factors and physical distancing are provided in the Supplementary Information (Supplementary Tables 1-10, 22-24, and 27-29).

Infection and fatality growth rates. Growth rate in infections was calculated as $\left(I_{t}-I_{t-1}\right) / I_{t-1} \times 100$. Exponential growth rate was calculated as the growth rate tailored to an exponential model of disease spread (Supplementary Information; $\left.r=\ln \left(I_{t+1} / I_{t}\right)\right)$. Fatality growth rate simply substitutes fatalities for infections. Calculated as such, growth rate is identical to growth rate in cases per capita because counties' populations did not vary during the sample period (see Supplementary Information for more details).

Reporting Summary. Further information on research design is available in the Nature Research Reporting Summary linked to this article.

\section{Data availability}

We are unable to share some of the data publicly because they are proprietary; to access these data please contact Unacast (https://www.unacast.com/covid19/ social-distancing-scoreboard\#get-started). The data are fully accessible upon agreeing to certain sharing restrictions and ethics guidelines. For instance, the data cannot be used for commercial purposes.

\section{Code availability}

Code for all analyses can be found at https://osf.io/u5pmw/?view_only=33f0691a7 e694276bef606cb3e22d141.

Received: 24 May 2020; Accepted: 17 September 2020; Published online: 2 November 2020

\section{References}

1. Perez-Truglia, R. \& Cruces, G. Partisan interactions: evidence from a field experiment in the United States. J. Polit. Econ. 125, 1208-1243 (2017).

2. Motyl, M., Iyer, R., Oishi, S., Trawalter, S. \& Nosek, B. A. How ideological migration geographically segregates groups. J. Exp. Soc. Psychol. 51, $1-14$ (2014)

3. Brady, W. J., Wills, J. A., Jost, J. T., Tucker, J. A. \& Van Bavel, J. J. Emotion shapes the diffusion of moralized content in social networks. Proc. Natl Acad. Sci. USA 114, 7313-7318 (2017).

4. Doherty, C., Kiley, J., \& Jameson, B. Partisanship and political animosity in 2016. Pew Research Center https://www.people-press.org/2016/06/22/ partisanship-and-political-animosity-in-2016/ (2016).

5. Garrett, R. K. Echo chambers online? Politically motivated selective exposure among internet news users. J. Comput. Mediat. Commun. 14, 265-285 (2009).

6. Iyengar, S. \& Krupenkin, M. Partisanship as social identity: implications for the study of party polarization. Forum 16, 23-45 (2019).

7. Mason, L. Uncivil Agreement: How Politics Became Our Identity (Univ. of Chicago Press, 2018).

8. McConnell, C., Margalit, Y., Malhotra, N. \& Levandusky, M. The economic consequences of partisanship in a polarized era. Am. J. Polit. Sci. 62, 5-18 (2018).

9. Frenda, S. J., Knowles, E. D., Saletan, W. \& Loftus, E. F. False memories of fabricated political events. J. Exp. Soc. Psychol. 49, 280-286 (2013).

10. Huber, G. A. \& Malhotra, N. Political homophily in social relationships: evidence from online dating behavior. J. Polit. 79, 269-283 (2017).

11. Data dive: the emotional cost of the 2016 election. Reuters https://www. reuters.com/article/us-datadive-election/data-dive-the-emotional-cost-of-the2016-election-idUSKBN15M1YC (2017).

12. Chen, M. K. \& Rohla, R. The effect of partisanship and political advertising on close family ties. Science 360, 1020-1024 (2018)

13. Bail, C. A. et al. Exposure to opposing views on social media can increase political polarization. Proc. Natl Acad. Sci. USA 115, 9216-9221 (2018).

14. Brady, W. J., Crockett, M. J. \& Van Bavel, J. J. The MAD model of moral contagion: the role of motivation, attention, and design in the spread of moralized content online. Perspect. Psychol. Sci. https://doi. org/10.1177/1745691620917336 (2020).

15. Martin, G. J. \& Yurukoglu, A. Bias in cable news: persuasion and polarization. Am. Econ. Rev. 107, 2565-2599 (2017).

16. Van Bavel, J. J. \& Pereira, A. The partisan brain: an identity-based model of political belief. Trends Cogn. Sci. 22, 213-224 (2018).

17. Van Bavel, J. J. et al. Using social and behavioral science to support COVID-19 pandemic response. Nat. Hum. Behav. 4, 460-471 (2020)

18. NBC News/Wall Street Journal Survey Datasets (Hart Research Associates/ Public Opinion Strategies, 2020); https://www.documentcloud.org/ documents/6810602-200149-NBCWSJ-March-Poll-Final-3-14-20-Release.html

19. Bird, R. \& Ritter, Z. Is the media creating division on COVID-19 health practices? Gallup https://news.gallup.com/poll/312749/ media-creating-division-covid-health-practices.aspx (2020).

20. Gadarian, S. K., Goodman, S. W. \& Pepinsky, T. B. Partisanship, health behavior, and policy attitudes in the early stages of the covid-19 pandemic. Preprint at SSRN https://doi.org/10.2139/ssrn.3562796 (2020).

21. Gollwitzer, A., Martel, C., Marshall, J., Höhs, J. M. \& Bargh, J. A. Connecting self-reported physical distancing to real-world behavior at the individual and us state level. Preprint at PsyArXiv https://doi.org/10.31234/ osf.io/kvnwp (2020).

22. Rosenfeld, D. L., Rothgerber, H. \& Wilson, T. Politicizing the COVID-19 pandemic: ideological differences in adherence to physical distancing. Preprint at PsyArXiv https://doi.org/10.31234/osf.io/k23cv (2020).

23. Fisher, R. J. Social desirability bias and the validity of indirect questioning. J. Consum. Res. 20, 303-315 (1993).

24. Maccoby, E. E. \& Maccoby, N. in Handbook of Social Psychology, Volume I Theory and Method (ed. Lindzey, G.) 449-487 (Addison-Wesley, 1954).

25. Bullock, J. G., Gerber, A. S., Hill, S. J. \& Huber, G. A. Partisan bias in factual beliefs about politics. Q. J. Polit. Sci. 10, 519-578 (2015). 
26. Ajzen, I. The theory of planned behavior. Organ. Behav. Hum. Decis. Process. 50, 179-211 (1991).

27. Anderson, R. M., Heesterbeek, H., Klinkenberg, D. \& Hollingsworth, T. D. How will country-based mitigation measures influence the course of the COVID-19 epidemic? Lancet 395, 931-934 (2020).

28. Gao, S. et al. Mobile phone location data reveal the effect and geographic variation of physical distancing on the spread of the COVID-19 epidemic. Preprint at arXiv https://arxiv.org/abs/2004.11430 (2020).

29. Hsiang, S. et al. The effect of large-scale anti-contagion policies on the COVID-19 pandemic. Nature 584, 262-267 (2020).

30. McGrail, D. J., Dai, J., McAndrews, K. M. \& Kalluri, R. Enacting national physical distancing policies corresponds with dramatic reduction in COVID-19 infection rates. PLoS ONE 15, e0236619 (2020).

31. Chinni, D. Uneven coronavirus spread leads to uneven partisan response. NBC News https://www.nbcnews.com/politics/meet-the-press/uneven-covidspread-leads-uneven-partisan-response-n1171491 (29 March 2020).

32. Painter, M. \& Qiu, T. Political beliefs affect compliance with COVID-19 physical distancing orders. Preprint at SSRN https://doi.org/10.2139/ ssrn.3569098 (2020).

33. Simonov, A., Sacher, S., Dubé, J. P. \& Biswas, S. The persuasive effect of Fox News: non-compliance with social distancing during the Covid-19 pandemic. NBER Working Paper No. 27237 (National Bureau of Economic Research, 2020).

34. Bursztyn L., Rao A., Roth C. \& Yanagizawa-Drott D. Misinformation during a pandemic. University of Chicago, Becker Friedman Institute for Economics Working Paper No. 2020-44https://bfi.uchicago.edu/wp-content/uploads/ BFI_WP_202044.pdf (2020).

35. Courtemanche, C., Garuccio, J., Le, A., Pinkston, J. \& Yelowitz, A. Strong social distancing measures in the United States reduced the COVID-19 growth rate. Health Aff. 39, 7 (2020).

36. Guan et al. Clinical characteristics of coronavirus disease 2019 in China. N. Engl. J. Med. 382, 1708-1720 (2020).

37. Lauer et al. The incubation period of coronavirus disease 2019 (COVID-19) from publicly reported confirmed cases: estimation and application. Ann. Intern. Med. 172, 577-582 (2020).

38. Linton et al. Incubation period and other epidemiological characteristics of 2019 novel coronavirus infections with right truncation: a statistical analysis of publicly available case data. J. Clin. Med. 9, 538 (2020).

39. Furukawa, N. W., Brooks, J. T. \& Sobel, J. Evidence supporting transmission of severe acute respiratory syndrome coronavirus 2 while presymptomatic or asymptomatic. Emerg. Infect. Dis. https://wwwnc.cdc.gov/eid/ article/26/7/20-1595_article (2020).

40. Johnson, D. B. \& Thompson, A. S. How non-pharmaceutical interventions, politics, race, and economic conditions impacted the rate of new infections of COVID-19. Preprint at OSF https://osf.io/yx7zs/ (2020).

41. Kim, D. D. \& Goel, A. Estimating case fatality rates of COVID-19. Lancet Infect. Dis. 20, 774-775 (2020).

42. Wilson, N., Kvalsvig, A., Barnard, L. T. \& Baker, M. G. Case-fatality risk estimates for COVID-19 calculated by using a lag time for fatality. Emerg. Infect. Dis. 26, 1339-1441 (2020).

43. Krull, J. L. \& MacKinnon, D. P. Multilevel modeling of individual and group level mediated effects. Multivar. Behav. Res. 36, 249-277 (2001).

44. Livadiotis, G. Statistical analysis of the impact of environmental temperature on the exponential growth rate of cases infected by COVID-19. PLoS ONE 15, e0233875 (2020).

45. Yuan, J., Li, M., Lv, G. \& Lu, K. Monitoring transmissibility and mortality of COVID-19 in Europe. Int. J. Infect. Dis. 95, 311-315 (2020).

46. Allcott, H. et al. Polarization and public health: partisan differences in social distancing during the coronavirus pandemic. NBER Working Paper No. 26946 (National Bureau of Economic Research, 2020).

47. Andersen, M. Early evidence on physical distancing in response to COVID-19 in the United States. Preprint at SSRN https://doi.org/10.2139/ ssrn.3569368 (2020).
48. Dyck, J. J. Political distrust and conservative voting in ballot measure elections. Polit. Res. Q. 63, 612-626 (2010).

49. Conway, L. Why conservatives and liberals are not experiencing the same pandemic. Heterodox: The Blog https://heterodoxacademy.org/ social-science-liberals-conservatives-covid-19/ (2020).

50. Smith, G. Fox News Nation. Preprint at SSRN https://ssrn.com/ abstract $=1766884$ (2020).

51. Haltiwanger, J. Video shows how Fox News rapidly shifted from downplaying coronavirus to taking it seriously as cases rose across the US. Business Insider https://www.businessinsider.com/coronavirus-video-shows-fox-news-shifttake-covid-19-seriously-2020-3 (2020).

52. Nail, P. R., McGregor, I., Drinkwater, A. E., Steele, G. M. \& Thompson, A. W. Threat causes liberals to think like conservatives. J. Exp. Soc. Psychol. 45, 901-907 (2009).

53. Janoff-Bulman, R. To provide or protect: motivational bases of political liberalism and conservatism. Psychol. Inq. 20, 120-128 (2009).

54. Doherty, C., Kiley, J. \& Jameson. Ebola worries rise, but most are 'fairly' confident in government, hospitals to deal with disease. Pew Research Center https://www.people-press.org/2014/10/21/ebolaworries-rise-but-most-are-fairly-confident-in-government-hospitals-todeal-with-disease/ (2014).

55. Cohen, G. L. Party over policy: the dominating impact of group influence on political beliefs. J. Pers. Soc. Psychol. 85, 808-822 (2003).

56. Macy, M., Deri, S., Ruch, A. \& Tong, N. Opinion cascades and the unpredictability of partisan polarization. Sci. Adv. 5, eaax0754 (2019).

57. Druckman, J. N., Klar, S., Krupnikov, Y., Levendusky, M. \& Ryan, J. B. The political impact of affective polarization: how partisan animus shapes COVID-19 attitudes. Preprint at PsyArXiv https://doi.org/10.31234/osf.io/ ztgpn (2020).

\section{Acknowledgements}

We thank J. Marshall, A. Deslatte, C. Routledge, and the NYU Social Identity \& Morality Lab for their helpful feedback. We also thank Unacast for sharing their data with us. P.P. was supported by the Swedish Research Council (no. 2016-06793). The funder had no role in study design, data collection and analysis, decision to publish or preparation of the manuscript. The authors received no specific funding for this work

\section{Author contributions}

A.G. developed the study concept and study design. Data preparation and acquisition were performed by A.G., C.M. and W.J.B. P.P., A.G., W.J.B., I.G.F., C.M. and E.D.K. completed the data analysis and interpretation. A.G., C.M. and P.P. drafted the manuscript. I.G.F., W.J.B., E.D.K. and J.J.V.B. provided critical revisions. All authors approved the final version of the manuscript for submission.

\section{Competing interests}

The authors declare no competing interests.

\section{Additional information}

Supplementary information is available for this paper at https://doi.org/10.1038/ s41562-020-00977-7.

Correspondence and requests for materials should be addressed to A.G. or J.J.V.

Peer review information Peer reviewer reports are available. Primary Handling Editor: Jamie, Horder

Reprints and permissions information is available at www.nature.com/reprints.

Publisher's note Springer Nature remains neutral with regard to jurisdictional claims in published maps and institutional affiliations.

(c) The Author(s), under exclusive licence to Springer Nature Limited 2020 


\section{Reporting Summary}

Nature Research wishes to improve the reproducibility of the work that we publish. This form provides structure for consistency and transparency in reporting. For further information on Nature Research policies, see our Editorial Policies and the Editorial Policy Checklist.

\section{Statistics}

For all statistical analyses, confirm that the following items are present in the figure legend, table legend, main text, or Methods section. $n / a \mid$ Confirmed

Х $\square$ The exact sample size $(n)$ for each experimental group/condition, given as a discrete number and unit of measurement

$\square$ \ A statement on whether measurements were taken from distinct samples or whether the same sample was measured repeatedly

$\square$ The statistical test(s) used AND whether they are one- or two-sided

$\square$ Only common tests should be described solely by name; describe more complex techniques in the Methods section.

$\square$ \ A description of all covariates tested

$\square$ \A description of any assumptions or corrections, such as tests of normality and adjustment for multiple comparisons

$\square$ A full description of the statistical parameters including central tendency (e.g. means) or other basic estimates (e.g. regression coefficient)

AND variation (e.g. standard deviation) or associated estimates of uncertainty (e.g. confidence intervals) $\bigotimes \square \begin{aligned} & \text { For null hypothesis testing, the test statistic (e.g. } F, t, r \text { ) with confidence intervals, effect sizes, degrees of freedom and } P \text { value noted } \\ & \text { Give } P \text { values as exact values whenever suitable. }\end{aligned}$

$\triangle \square$ For Bayesian analysis, information on the choice of priors and Markov chain Monte Carlo settings

$\square$ For hierarchical and complex designs, identification of the appropriate level for tests and full reporting of outcomes

$\square$ Estimates of effect sizes (e.g. Cohen's d, Pearson's $r$ ), indicating how they were calculated

Our web collection on statistics for biologists contains articles on many of the points above.

\section{Software and code}

\section{Policy information about availability of computer code}

Data collection The data were collected by Unacast.

Data analysis See code availability statement.

For manuscripts utilizing custom algorithms or software that are central to the research but not yet described in published literature, software must be made available to editors and reviewers. We strongly encourage code deposition in a community repository (e.g. GitHub). See the Nature Research guidelines for submitting code \& software for further information.

\section{Data}

Policy information about availability of data

All manuscripts must include a data availability statement. This statement should provide the following information, where applicable:

- Accession codes, unique identifiers, or web links for publicly available datasets

- A list of figures that have associated raw data

- A description of any restrictions on data availability

We are unable to publicly share the included data as it is proprietary. To access the included data please contact Unacast directly. The data is fully accessible upon agreeing to certain sharing restrictions and ethics guidelines. For instance, the data cannot be used for commercial purposes and can only be used for academic/ research purposes. The data availability statement conveys this information and provides a direct link via which to contact Unacast. 


\section{Field-specific reporting}

Please select the one below that is the best fit for your research. If you are not sure, read the appropriate sections before making your selection. $\square$ Life sciences $\quad \square$ Behavioural \& social sciences $\quad \square$ Ecological, evolutionary \& environmental sciences

For a reference copy of the document with all sections, see nature.com/documents/nr-reporting-summary-flat.pdf

\section{Behavioural \& social sciences study design}

All studies must disclose on these points even when the disclosure is negative.

\begin{tabular}{|c|c|}
\hline Study description & Quantitative Data \\
\hline Research sample & $\begin{array}{l}\text { Smart-phone users around the United States. It is unclear whether this sample is representative. Demographic information is } \\
\text { unknown for privacy reasons. }\end{array}$ \\
\hline Sampling strategy & $\begin{array}{l}\text { The data was collected and shared with us by Unacast. As such, the sample size was determined by Unacast rather than us. Given } \\
\text { that the data is the geo-location of } 15 \text { millions smartphone users a day, we believe the sample size allows for accurate estimates of } \\
\text { counties' degree of physical distancing. }\end{array}$ \\
\hline Data collection & The data was collected by Unacast using thousands of opt-in consent mobile Apps. \\
\hline Timing & March 9th to May 29th, 2020 \\
\hline Data exclusions & We excluded counties with a population less than 2,000 people from the analyses. \\
\hline Non-participation & N/A \\
\hline Randomization & Participants were not allocated into groups \\
\hline
\end{tabular}

\section{Reporting for specific materials, systems and methods}

We require information from authors about some types of materials, experimental systems and methods used in many studies. Here, indicate whether each material, system or method listed is relevant to your study. If you are not sure if a list item applies to your research, read the appropriate section before selecting a response.

Materials \& experimental systems Methods

\begin{tabular}{|c|c|}
\hline$n / a$ & Involved in the study \\
\hline & $\square$ Antibodies \\
\hline & Eukaryotic cell lines \\
\hline & Palaeontology and archaeology \\
\hline & Animals and other organisms \\
\hline & $\square$ Human research participants \\
\hline & $\square$ Clinical data \\
\hline & $\square$ Dual use research of concern \\
\hline
\end{tabular}

n/a $\mid$ Involved in the study

\ $\square$ ChIP-seq

Х $\square$ Flow cytometry

$\bigotimes \square$ MRI-based neuroimaging 